\title{
microRNA-155 is downregulated in gastric cancer cells and involved in cell metastasis
}

\author{
CHENG-LONG LI $^{1}{ }^{*}$, HUI NIE $^{1,2^{*}}$, MING WANG ${ }^{1}$, LI-PING SU ${ }^{1}$, JIAN-FANG LI ${ }^{1}$, \\ YING-YAN YU ${ }^{1}$, MIN YAN ${ }^{1}$, QIN-LONG QU ${ }^{1}$, ZHENG-GANG ZHU ${ }^{1}$ and BING-YA LIU ${ }^{1}$ \\ ${ }^{1}$ Shanghai Key Laboratory of Gastric Neoplasms, Department of Surgery, Shanghai Institute of Digestive Surgery, \\ Ruijin Hospital, Shanghai Jiao Tong University School of Medicine, Shanghai 200025; ${ }^{2}$ School of Biomedical \\ Engineering and Med-X Research Institute, Shanghai Jiao Tong University, Shanghai 200025, P.R. China
}

Received November 21, 2011; Accepted February 13, 2012

DOI: $10.3892 /$ or.2012.1719

\begin{abstract}
RNA-155 (miR-155), an important multifunctional microRNA, has been implicated in the development of multiple solid tumors, yet, its role in gastric cancer cells has not been fully elucidated. In this study, we find that miR-155 was significantly downregulated in gastric cancer cell lines compared with an immortalized gastric epithelial cell line (GES-1). Overexpression of miR-155 in SGC-7901 and MKN-45 gastric cancer cells dramatically suppressed cell migration, invasion and adhesion in vitro. Overexpression of miR-155 significantly reduced the protein levels of SMAD2 and repressed the activity of a luciferase reporter containing one of the two predicted miR-155 binding sites in SMAD2 3'-UTR, indicating that SMAD2 may be a miR-155 target gene. miR-155 expression was also remarkably restored by a DNA demethylating agent (5-Aza-2-deoxycytidine) in SGC-7901 and MKN-45 gastric cancer cells. Taken together, these data suggest that miR-155 may function as a tumor suppressor to regulate gastric cancer cell metastasis by targeting SMAD2, and its downregulation in gastric cancer cells may be partly ascribed to DNA methylation.
\end{abstract}

\section{Introduction}

Gastric cancer remains the fourth most common malignancy and the second leading cause of cancer-related death in both genders worldwide, despite a substantial decrease in its rate

Correspondence to: Professor Bing-Ya Liu, Shanghai Key Laboratory of Gastric Neoplasms, Department of Surgery, Shanghai Institute of Digestive Surgery, Ruijin Hospital, Shanghai Jiao Tong University School of Medicine, Shanghai 200025, P.R. China

E-mail: liubyrj@yahoo.com.cn

"Contributed equally

Key words: microRNA-155, gastric cancer, SMAD2, metastasis, DNA methylation
$(1,2)$. In 2008 , more than $70 \%$ of new cases $(713,000$ cases $)$ occurred in developing countries, and half the world total occured in Eastern Asia (mainly in China) (2). Despite a steady decline in mortality, the prognosis of patients with advanced gastric cancer is still very disappointing (3), mainly due to poor understanding of the mechanisms underlying its development.

microRNAs (miRNAs) are endogenous, small (18-25 nucleotides), non-coding RNAs that play important roles in gene expression by targeting 3'UTR of cellular transcripts, resulting mRNA cleavage or translational inhibition $(4,5)$. By these mechanisms, miRNAs have been shown to be involved in mediating processes in tumorigenesis such as cell cycle regulation, differentiation, apoptosis, invasion and metastasis $(6,7)$. Dysregulated expression of miRNAs is now considered as a common characteristic of all human tumors (8). The aberrant expression of miRNAs in human cancers and their role in tumor formation might define miRNAs as oncogenes or tumor suppressors.

miR-155, processed from an exon of a non-coding RNA transcribed from the B-cell integration cluster (BIC), has been a typical multifunctional miRNA $(9,10)$. Accumulating evidence has demonstrated that miR-155 is involved in numerous biological processes including haematopoiesis, inflammation, immunity and carcinogenesis $(10,11)$. Several lines of evidence indicate that dysregulation of miR-155 is linked to various solid tumors, including breast, colon, cervical, thyroid, pancreatic and lung cancer (12-18). The frequently detected overexpression of miR-155 in these tumors indicates a major role of an oncogene; however, possible tumor suppressor functions have also been reported (19-21). Although miR-155 has been found to be dysregulated in several kinds of cancer, its role in gastric cancer tumorigenesis has not yet been defined. Analyses in our laboratory of miRNA expression profile of gastric cancer cell lines have shown that miR-155 was one out of 146 downregulated miRNAs. Here, we further demonstrate a general decrease in miR-155 expression level in gastric cancer cells. Ectopic expression of miR-155 significantly inhibited the migration, invasion and adhesion of gastric cancer cells in vitro. By gain-of-function examination, SMAD2 was identified as a significant target of miR-155, contributing to its anti-oncomiR effect. We also explored the underlying mechanisms of reduced miR-155 expression in gastric cancer cells. 


\section{Materials and methods}

Cell lines. Two gastric cancer cell lines, MKN-45 and MKN-28, were obtained from the Japanese Cancer Research Resources Bank, SGC-7901, NCI-N87 and AGS were purchased from Shanghai Institutes for Biological Sciences, Chinese Academy of Sciences, and the other gastric cancer cell lines were obtained from the American Type Culture Collection. GES-1, an immortalized gastric epithelial cell line, was a gift from Professor Feng Bi (Huaxi Hospital, Sichuan University, China). Human embryonic kidney cell line 293T (HEK 293T) was preserved in our institute. All the gastric cancer cell lines and GES-1 were maintained in RPMI-1640 supplemented with $10 \%$ heat-inactivated fetal bovine serum in a humidified cell incubator having an atmosphere of $5 \% \mathrm{CO}_{2}$ at $37^{\circ} \mathrm{C}$. HEK $293 \mathrm{~T}$ was maintained in Dulbecco's modified Eagle's medium (DMEM) supplemented with $10 \%$ heat-inactivated fetal bovine serum in the same condition. Exponentially growing cells were used for experiments.

RNA isolation and $q R T-P C R$. Total RNA was extracted using a mirVana $^{\mathrm{TM}}$ miRNA Isolation Kit (Applied Biosystems, Foster City, CA, USA) according to the manufacturer's instructions. Concentrations and purity of the RNA samples were assayed by electrophoresis and spectrophotometric methods. Mature miR-155 expression was assessed by qRT-PCR according to the TaqMan ${ }^{\circledR}$ microRNA Assays protocol (Applied Biosystems). U6 small nuclear RNA (RNU6B; Applied Biosystems) was used for normalization. All reactions were performed in triplicate. Expression levels of miRNAs were analysed by the comparative CT method $\left(2^{-\Delta \mathrm{CT}}\right)$.

Transfection of miRNA mimics. miR-155 mimics (dsRNA oligonucleotides) and negative control mimics (control) were purchased from Shanghai GenePharma Co., Ltd., Shanghai, China. Gastric cancer cells were seeded into 6-well plates the day before transfection to ensure $50 \%$ cell confluence at the moment of transfection. Transfection of miRNA mimics was performed at $100 \mathrm{nM}$ final concentration using Lipofectamine 2000 (Invitrogen, Carlsbad, CA, USA). qRT-PCR and western blot analysis were carried out at $48 \mathrm{~h}$ post-transfection. Transfection efficiency was monitored by the transfection of GMR-miR ${ }^{\mathrm{TM}}$ microRNA FAM-labeled microRNA mimics control (Shanghai GenePharma Co., Ltd.).

Scratch wound healing assays. Cells were seeded on 6-well plates at $60 \%$ confluency for $12 \mathrm{~h}$ before transfection. After transfection with miRNA mimics, cells were incubated at $37^{\circ} \mathrm{C}$ until they reach $100 \%$ confluence to form a monolayer. The cell layer was scratched with a pipette tip and then grown under normal conditions after washing with culture medium. The wound closing process was observed for $48 \mathrm{~h}$. Photographs were taken every $6 \mathrm{~h}$ (under x100 magnification).

Cell migration and invasion assay. Cell migration and invasion assays were performed using transwell chambers (8 $\mu \mathrm{m}, 24$-well insert; Corning, Lowell, MA, USA). At $24 \mathrm{~h}$ post-transfection with miRNA mimics $(100 \mathrm{nM})$, cells were incubated in serum-free medium for $24 \mathrm{~h}$, and then $2 \times 10^{4}$ cells in $200 \mu \mathrm{l}$ serum-free medium were added to the upper chamber. For invasion assay, the insert membranes were coated with diluted Matrigel (BD Biosciences, Franklin Lakes, NJ, USA). For migration assay, the insert membranes were not coated with Matrigel but were cultured under the same conditions. A volume of $0.6 \mathrm{ml}$ medium containing $10 \%$ FBS was added to the lower chambers. Cells were incubated at $37^{\circ} \mathrm{C}, 5 \% \mathrm{CO}_{2}$ for $48 \mathrm{~h}$. After removing the media from the inserts, non-migrating cells from the interior of the inserts were removed with cotton-tipped swabs. Cells that migrated to the bottom of the membranes were stained with crystal violet $(0.1 \%$ in methanol) for $20 \mathrm{~min}$. The stained cells were counted under the inverted microscope and photographed with x100 magnification. At least three randomly selected fields were counted and the average number was taken. The data were shown as the means $\pm \mathrm{SD}$.

Cell adhesion assays. Cell adhesion was measured using CytoSelect $^{\mathrm{TM}}$ Cell Adhesion Assay (Cell Biolabs, San Diego, CA, USA). Briefly, at $24 \mathrm{~h}$ post-transfection with miRNA mimics, cells were harvested and resuspended in serum-free RPMI-1640 with a concentration of $5 \times 10^{5}$ cells $/ \mathrm{ml}$. For each group, four Collagen IV coated wells and one BSA coated well were used. A volume of $150 \mu \mathrm{l}$ cell suspension was added to the inside of each well. The plate was then incubated at $37^{\circ} \mathrm{C}$, $5 \% \mathrm{CO}_{2}$ for $90 \mathrm{~min}$. Each well was then gently washed four times with $250 \mu \mathrm{l}$ of PBS. Cells were stained with $200 \mu \mathrm{l}$ of cell stain solution (Cell Biolabs) per well for $10 \mathrm{~min}$ at room temperature. The stain was then removed and the cells were washed five times with $500 \mu$ l deionized water. After allowing the cells to air dry for $25 \mathrm{~min}, 200 \mu \mathrm{l}$ of extraction solution (Cell Biolabs) was added to each well and incubated for $10 \mathrm{~min}$ on an orbital shaker. Absorbance of the extracted samples was measured at $560 \mathrm{~nm}$ by a Tecan Safire 2 microplate reader (Tecan, Männedorf, Switzerland). Results were obtained by subtracting absorbance of the negative controls.

Plasmid construction and luciferase assays. As previously described (22), complementary DNA oligonucleotides containing the two putative miR-155 target sites within the 3'UTR of human SMAD2 mRNA were synthesized with flanking SpeI and HindIII restriction enzyme digestion sites using the following primers: 5'-CTAGTGGTGAGATTTTT TTTCTCTGATGGCATTAACTTTGTAATGCAATATGAT GA-3' (WT1-sense), 5'-AGCTTCATCATATTGCATTACA AAGTTAATGCCATCAGAGAAAAAAAATCTCACCA-3' (WT1-antisense), 5'-CTAGTAAAGGTGGACAGTTTT CCTAGCATTAAAAGAAAGCCATTTGAGA-3' (WT2sense), 5'-AGCTTCTCAAA-TGGCTTTCTTTTAATGC TAGGAAAACTGTCCACCTTTA-3' (WT2-antisense). Each strand of oligonucleotides $(13 \mu \mathrm{l})(100 \mathrm{nM})$ was added to $3 \mu \mathrm{l}$ of the 10X annealing buffer (100 mM Tris- $\mathrm{HCl}, \mathrm{pH} 8.0 ; 10 \mathrm{mM}$ EDTA, pH 8.0; $1 \mathrm{M} \mathrm{NaCl}$ ) and incubated at $90^{\circ} \mathrm{C}$ for $5 \mathrm{~min}$ and then at room temperature for $30 \mathrm{~min}$. The annealed oligonucleotides were digested with SpeI and HindIII and cloned into the pMIR-Report Luciferase vector (Applied Biosystems). To identify direct targeting of miR-155, the vectors were mutated in the two putative miR-155 binding sites using primers as follows: 5'-CTAGTGGTGAGATTTTTTTTCTCTGATGG GAATTACTTTGTAATGCAATATGATGA-3' (MUT1sense), 5'-AGCTTCATCATATTGCATTACAAAGTAATT 
CCCATCAGAGAAAAAAAATCTCACCA-3' (MUT1antisense), 5'-CTAGTAAAGGTGGACAGTTTTCCTACCT TAATAAGAAAGCCATTTGAGA-3' (MUT2-sense), 5'-AG CTTCTCAAATGGCTTTCTTATTAAGGTAGGAAAACT GTCCACCTTTA-3' (MUT2-antisense). HEK 293T cells were seeded in 24-well plates $24 \mathrm{~h}$ prior to transfection. In each well, the cells were transfected with $500 \mathrm{ng}$ of pMIR-report3'UTR construct, together with $10 \mathrm{ng}$ of the pRL-TK vector (Promega, Madison, WI, USA) containing Renilla luciferase and $60 \mathrm{pmol}$ of the miRNA mimics. Cells were harvested $48 \mathrm{~h}$ after transfection. Luciferase activity was measured using the dual-luciferase reporter assay system (Promega) and normalized to Renilla luciferase activity.

Western blot analysis. SGC-7901 or MKN45 cancer cells were transfected with microRNA mimics in 6-well plates. Forty-eight hours after transfection, protein from the cells was extracted by RIPA buffer (Beyotime, Nantong, China) in the presence of protease inhibitors PMSF (Beyotime). The protein concentration of the lysates was assayed using a BCA Protein Assay Kit (Pierce, Appleton, WI, USA). Equal amounts of protein $(50 \mu \mathrm{g})$ were resolved by $10 \%$ SDS-PAGE and then transferred to PVDF membranes. After blocked with 5\% non-fat milk in TBST buffer (Trisbuffered saline plus $0.1 \%$ Tween-20), the membranes were hybridized with primary antibody, followed by incubation with specific HRP-conjugated secondary antibody (Abcam, Hong Kong). Finally, the proteins were visualized by an enhanced chemiluminescence detection system (Millipore, Billerica, MA, USA). Mouse monoclonal anti-SMAD2 (1:1000, Cell Signaling Technology, Danvers, MA, USA) was used. Monoclonal anti-GAPDH (1:5000, Abcam) served as a loading control.

5-Aza-2-deoxycytidine treatment. SGC-7901 or MKN45 cancer cells were seeded in 6-well plates and treated with 10 mM 5-Aza-2-deoxycytidine (5-Aza-dC; Sigma, St. Louis, MO, USA) for $96 \mathrm{~h}$ per well; the control group was treated with PBS. After treatment, the cells were harvested and total RNA was extracted. The level of miR-155 expression in treated and control cells was measured by qRT-PCR as described above.

Statistical analysis. Results were expressed as means \pm standard deviation from at least three separate experiments. All statistical analyses were performed using the SPSS 15.0 software (SPSS Inc., Chicago, IL, USA). Statistical significance between two different groups was determined by Student's t-test. A two-tailed value of $\mathrm{P}<0.05$ was considered statistically significant.

\section{Results}

Downregulation of miR-155 in gastric cancer cells. First, we screened miR-155 and SMAD2 expression in several gastric cancer cell lines, which were compared with GES-1 by qRT-PCR and western blot analysis. As shown in Fig. 1, miR-155 was significantly downregulated in all gastric cancer cell lines compared with GES-1, while SMAD2 showed an upregulated expression.

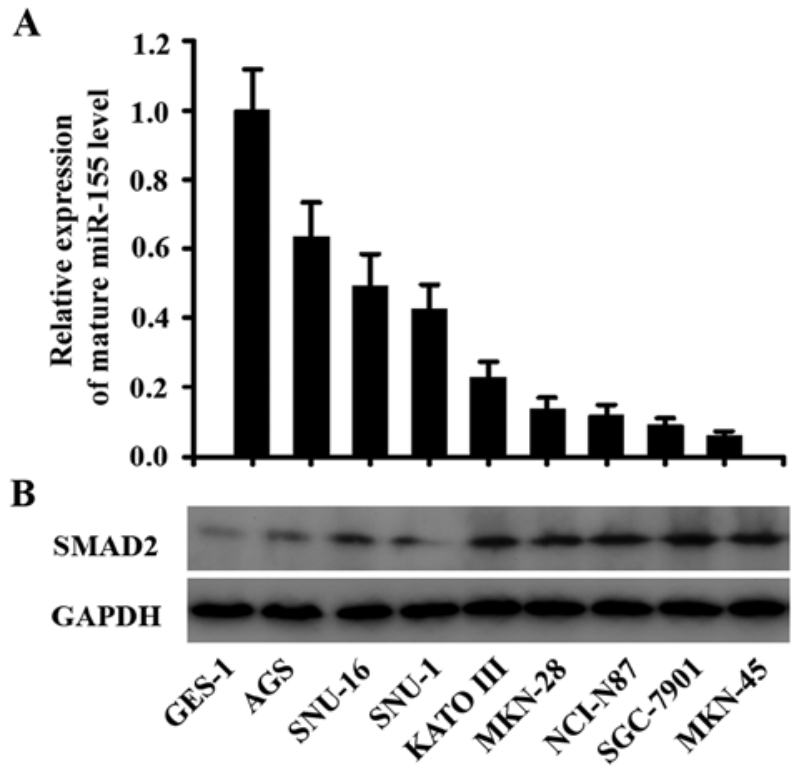

Figure 1. Various expression levels of miR-155 and SMAD2 on eight gastric cell lines and one immortalized gastric epithelial cell line (GES-1). (A) Downregulation of miR-155 in gastric cancer cell lines was carried out by qRT-PCR. (B) Western blot analysis of SMAD2 upregulation in gastric cancer cell lines. GAPDH was used as loading control.

Functions of miR-155 on gastric cancer cells. miR-155 may play the role of tumor suppressor, considering that it was significantly downregulated in gastric cancer. For this reason, we examined the effects of miR-155 overexpression in SGC-7901 and MKN-45 cells, both of which had very low level of endogenous miR-155. Synthetic miR-155 mimics were transfected into gastric cancer cells to restore miR-155 expression, and its restoration was confirmed by qRT-PCR.

First, we examined cell migration and invasive ability by scratch wound healing assay, transwell migration and transwell invasion assay. As shown in Fig. 2, cells transfected with miR-155 closed the scratch-wounds more slowly than cells transfected with control. The mean wound distances of the experimental sample and the control at $48 \mathrm{~h}$ were significantly different in MKN-45 cells $(358.8 \pm 87.1 \mathrm{~mm}$ vs. $133.4 \pm 48.6 \mathrm{~mm}, \mathrm{P}<0.001)$. Scratch healing results observed in SGC-7901 cells at $48 \mathrm{~h}$ also showed the same tendency. $(486.3 \pm 94.8 \mathrm{~mm}$ vs. $229.7 \pm 39.2 \mathrm{~mm}, \mathrm{P}<0.001)$. Transwell migration results showed that miR-155 overexpression significantly decreased the amount of migrated cells in both cell lines $(86.3 \pm 13.7$ vs. $145.6 \pm 20.2$ in SGC-7901 cells, $\mathrm{P}<0.01$, $100.4 \pm 17.1$ vs. $169.5 \pm 25.8$ in MKN-45 cells, $\mathrm{P}<0.01$, Fig. $3 \mathrm{~A}$ and $\mathrm{B})$. Cell invasion assays also indicated that cells overexpressing miR-155 showed lower invasive capacity compared with cells transfected with control $(52.3 \pm 9.8$ vs. $97.4 \pm 17.2$ in SGC-7901 cells, $\mathrm{P}<0.01,82.7 \pm 11.4$ vs. $133.9 \pm 23.1$ in $\mathrm{MKN}-45$ cells, $\mathrm{P}<0.01$, Fig. $3 \mathrm{~A}$ and $\mathrm{C}$ ).

Next, we examined changes of cell adhesion capacity to collagen type IV. In accordance with cell migration and invasion differences, ectopic expression of miR-155 in the gastric cancer cells reduced their attachment to collagen type IV $(0.414 \pm 0.039$ vs. $0.669 \pm 0.041$ in SGC-7901 cells, $\mathrm{P}<0.01$, $0.295 \pm 0.028$ vs. $0.501 \pm 0.036$ in $\mathrm{MKN}-45$ cells, $\mathrm{P}<0.01$, Fig. 3D). Thus, it was more difficult for them to shed from 


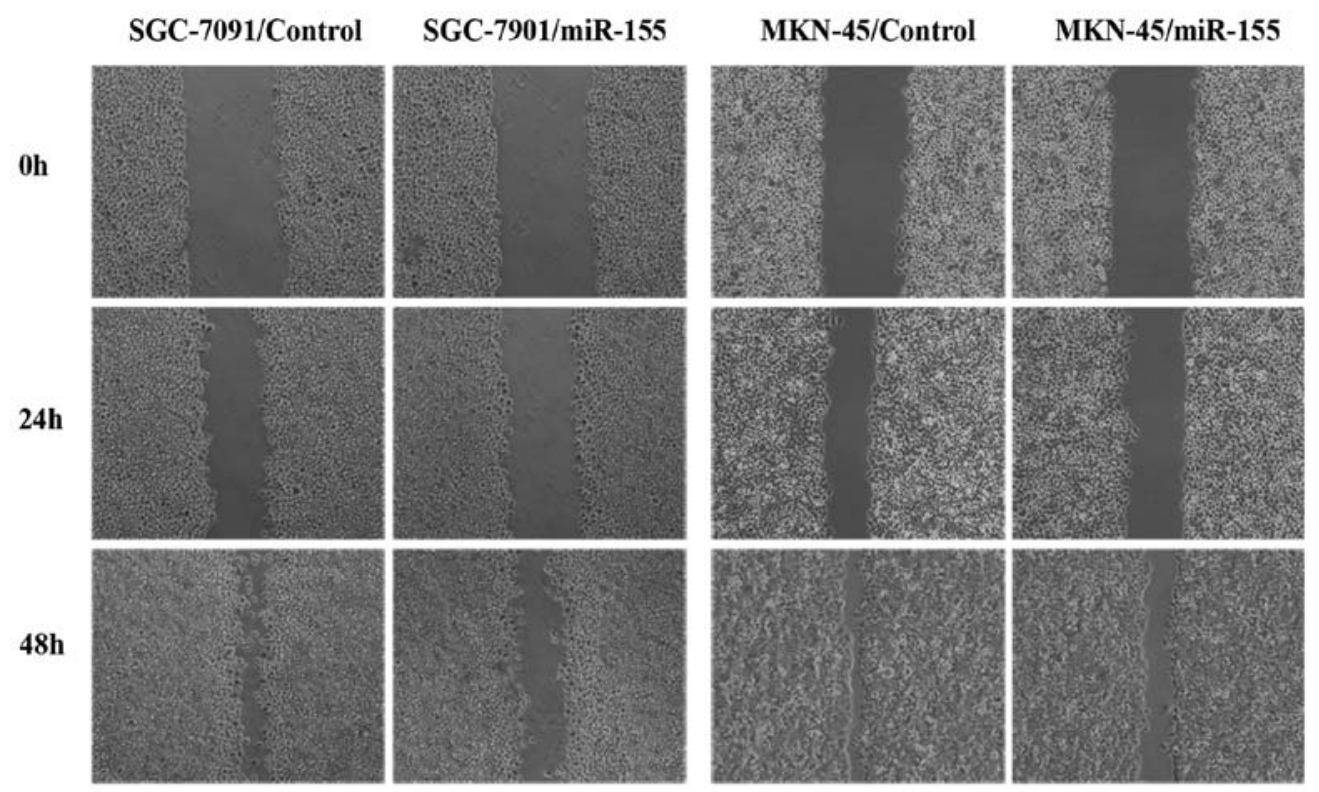

Figure 2. Scratch wound healing assay shows that miR-155 negatively regulated gastric cancer cell migration ability in vitro. Confluent cell monolayers were wounded with a pipette tip. Wound closure was monitored by microscopy at the indicated times. By wound distance analysis, cell migration ability was suppressed by miR-155 overexpression.

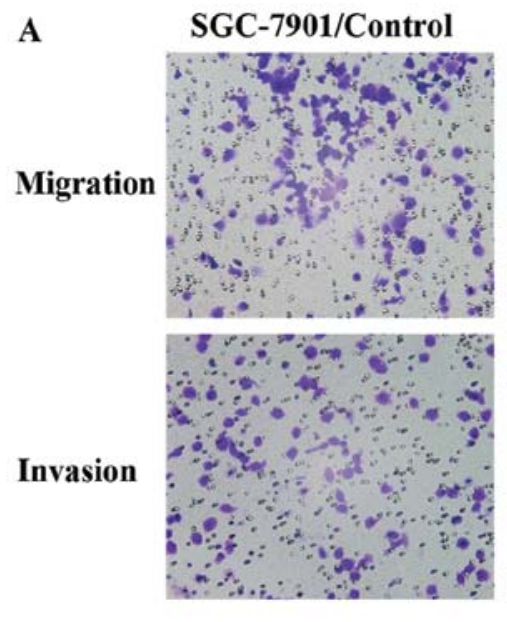

B

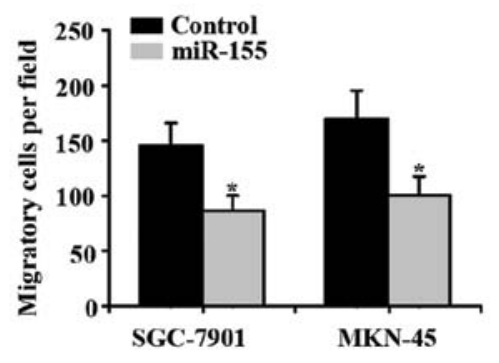

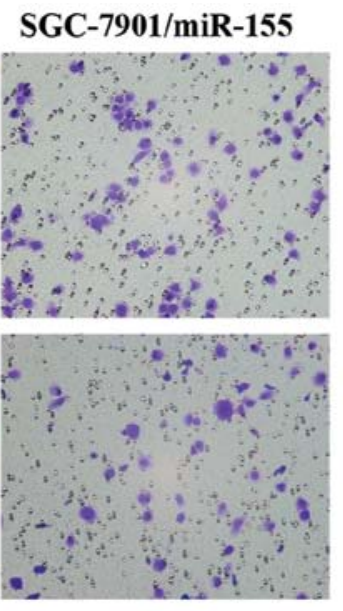

C

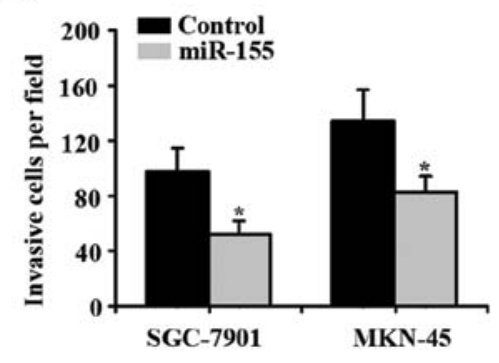

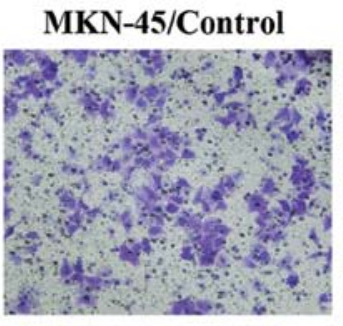

MKN-45/miR-155
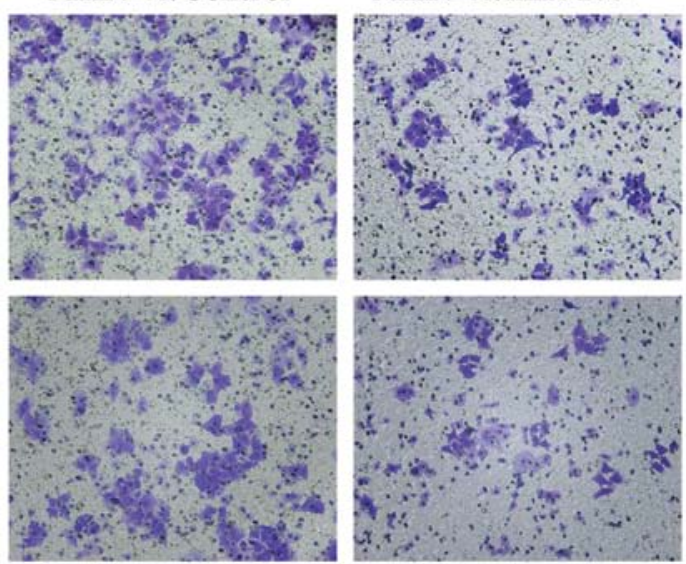

D

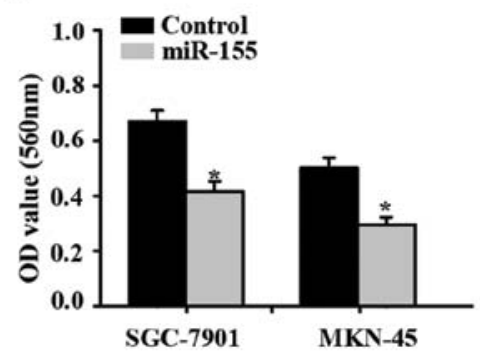

Figure 3. miR-155 inhibits migration, invasion and adhesion of gastric cancer cells in vitro. (A) Representative photographs of migratory cells on the membrane of transwell chambers in migration and invasion assays (magnification, $\mathrm{x} 100$ ). (B) Average migratory cell number of triplicate experiments \pm SD. ("P<0.01). (C) Average invasive cell number of triplicate experiments \pm SD; ( $" \mathrm{P}<0.01)$. (D) miR-155 reduced gastric cancer cell attachment to collagen type IV. Values represent mean optical density $(\mathrm{OD}) \pm \mathrm{SD}$ of three independent experiments $\left({ }^{*} \mathrm{P}<0.01\right)$.

primary sites and to migrate. Cell migration, invasion and adhesion are essential processes in cancer metastasis. These results display an inhibitory role for miR-155 in gastric cancer metastasis in vitro.
miR-155 targetting 3'UTR of the SMAD2 gene. Since miR-155 has a pivotal function of metastasis in gastric cancer cells, we searched for its putative target genes with potential metastatic functions using online search tools (MicroCosm, 
$\mathbf{A}$

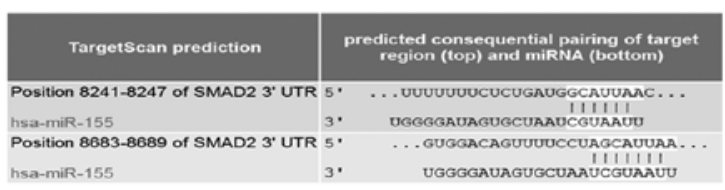

B

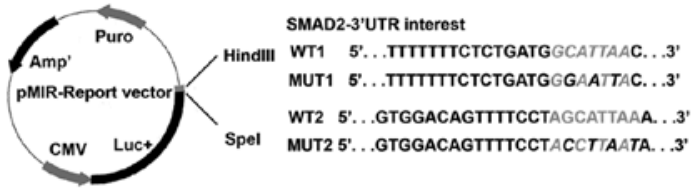

C

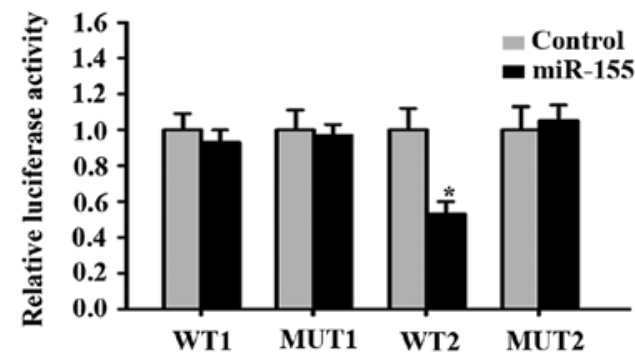

D

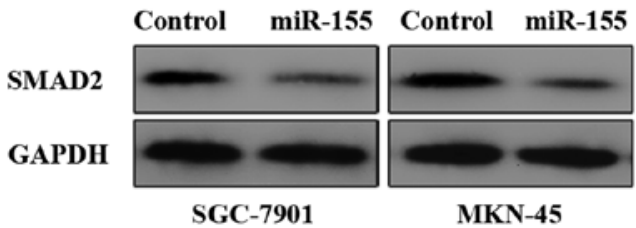

Figure 4. SMAD2 is a validated target of miR-155. (A) Putative binding sites of miR-155 in the SMAD2 3'UTR (white sequences) predicted by TargetScan (B) Construction of the reporter plasmid. WT1, MUT1, WT2 and MUT2, respectively, were inserted into downstream of luciferase of pMIR-reporter vector. (C) Dual luciferase report assays were performed on HEK 293T cells Each bar represents mean values \pm SD from three independent experiments (*P<0.01). (D) Forty-eight hours after miR-155 mimics or control transfection on SGC-7901 and MKN-45 cells, SMAD2 protein level was significantly reduced in miR-155 transfected cells by western blot analysis.

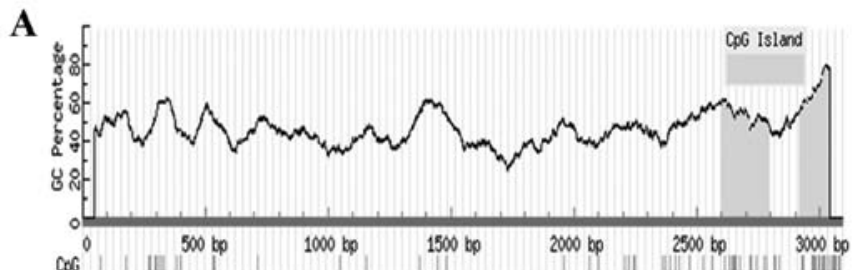

B

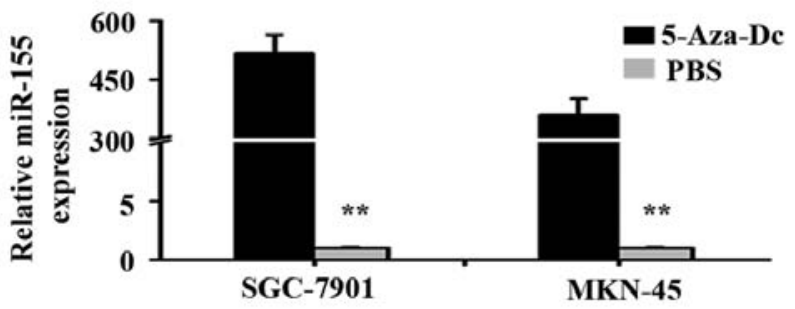

Figure 5. DNA methylation is involved in the regulation of miR-155 expression. (A) MethPrimer program revealed that the promoter region of miR-155 contained two CpG islands. (B) miR-155 expression was remarkably increased after 5-Aza-dC treatment in SGC-7901 and MKN-45 cells by qRT-PCR assays $\left({ }^{* *} \mathrm{P}<0.001\right)$.

TargetScan and Microrna.org). Among these genes, SMAD family member 2 (SMAD2), one of the mediators of TGF- $\beta$ signaling, was predicted to have two putative miR-155 binding sites within its 3'UTR by both TargetScan and Microrna.org. In addition, SMAD2 was validated as a target gene of miR-155 in macrophages (23). Therefore, we further confirmed whether SMAD2 was also the authentic target gene of miR-155 in gastric cancer.

miR-155 was predicted to target the end of SMAD2 3'UTR at two distinct sites, which were located at position 8241-8247 (7-mer) and position 8683-8689 (8-mer), respectively (Fig. 4A). To validate the direct binding of miR-155 to SMAD2 3'UTR, we performed dual luciferase report assays in HEK 293T cells. We cloned two regions of 3'UTR each containing one putative miR-155 binding site into the pMIR-Report luciferase vector (WT1 for the 7-mer site and WT2 for the 8-mer site) (Fig. 4B). The reporter constructs harboring mutation of the miR-155 target sites were generated similarly and named as MUT1 (mutation of the 7-mer site) and MUT2 (mutation of the 8-mer site) (Fig. 4B). The luciferase reporter constructs were transfected into HEK 293T cells, along with miRNA mimics and pRL-TK. Luciferase activites were then measured. The luciferase activity of WT2 reporter transfected with miR-155 mimics was significantly decreased compared with control $(\mathrm{P}<0.01)$, while the luciferase activity of the WT1 reporter was not interfered with after transfection with miR-155 mimics compared with control $(\mathrm{P}>0.05)$. Moreover, MUT2 clearly abrogated the repression of luciferase activity caused by miR-155 overexpression (Fig. 4C). These data indicate that miR-155 may target SMAD2 gene through the 8-mer seeding region of 3'UTR.

Furthermore, western blot assays showed that an enforced miR-155 expression led to a reduction of SMAD2 protein both in SGC-7901 and MKN-45 gastric cancer cells (Fig. 4D). Moreover, a significant inverse correlation between miR-155 expression and SMAD2 protein level was found in gastric cancer cell lines (Fig. 1). Taken together, our data suggest that miR-155 may attenuate the expression of SMAD2 by directly targeting the 3'UTR of SMAD2.

Regulation of miR-155 expression in gastric cancer cells. We next investigated why miR-155 expression was downregulated in gastric cancer cells. Bioinformatic analysis of the BIC promoter region by MethPrimer program (http://www.urogene. org/methprimer) revealed that it contained two putative $\mathrm{CpG}$ islands (Fig. 5A). To further establish that DNA methylation is involved in the regulation of miR-155 expression, we treated two gastric cancer cell lines (SGC-7901 and MKN-45) with $10 \mu \mathrm{M}$ 5-Aza-dC (a DNA-demethylating agent) or PBS for $96 \mathrm{~h}$. The expression levels of miR-155 were remarkably restored (after treatment with 5-Aza-dC for SGC-7901 cells, a 517-fold upregulation; and for MKN-45 cells, a 360-fold upregulation; Fig. 5B). The result suggests that the silencing of miR-155 expression in gastric cancer may be partly ascribed to DNA methylation.

\section{Discussion}

Although miRNA signatures of gastric cancer have been established, knowledge of the role of deregulated miRNAs in gastric carcinogenesis remains largely unknown. The data presented here show a role of miR-155 in gastric cancer. 
miR-155 locates within the BIC gene on chromosome 21 in humans (24). To date, miR-155 has been widely involved in different kinds of cancer, cardiovascular diseases and viral infections. High levels of miRNA-155 have been found in neoplastic diseases in both hematopoietic malignancies (i.e. Hodgkin's lymphoma, B-CLL, B-cell lymphomas, AML and CML) and solid tumors (e.g. breast, colon, cervical, thyroid, pancreatic and lung cancer). Relatively high levels of miR-155 and repressing the expression of tumor suppressors had been found in different types of cancers, hence miR-155 was considered oncogenic. On the other hand, a very low or null of miR-155 expression was reported in most non-Hodgkin lymphoma subtypes including Burkitt lymphoma (25). miR-155 was also found to be expressed in healthy pancreas and essentially absent in endocrine pancreatic tumors (26). Decreased levels of this miRNA were also described in ovarian cancer (27), melanoma $(20,21)$, human papillomavirus (HPV)-positive cases of squamous cell carcinoma of the head and neck (SCCHN) (28) and EBV-infection of diffuse large B-cell lymphoma (DLBCL) (29). In these malignancies, a tumor suppressive function of miR-155 has been therefore suggested. Up to now, little has been known about the role of miR-155 in gastric cancer.

In this study, we first demonstrate that miR-155 expression was repressed in gastric cancer cells compared with an immortalized gastric mucosa cell line and that it probably functioned as an anti-oncomiR. Overexpression of miR-155 in gastric cancer cells significantly inhibited cell migration, invasion and adhesion in vitro. Cell motility and invasion are essential features of the metastatic process. That is, miR-155 exhibited an attenuated impact on gastric cancer cell metastasis in vitro. Further studies based on in vivo and clinical evidence would be required to fully understand the role of miR-155 in gastric cancer metastasis.

To investigate the molecular mechanism underlying miR-155 function, we searched for its direct target genes using bioinformatic analysis and found that SMAD2 has two putative miR-155 binding sites (7-mer and 8-mer) within its 3'UTR. We showed that only the 8-mer site contributed to miR-155 repressing actions on the 3'UTR of SMAD2; similar results were obtained in a recent study on miR-155 targeting SMAD2 in macrophages (23). SMAD2 is the primary intracellular signaling pathway downstream of transforming growth factorbeta (TGF- $\beta$ ) (30). TGF- $\beta$ can act as an oncogenic factor inducing proliferation, angiogenesis, invasion and metastasis (31). High TGF- $\beta$-Smad activity was present in aggressive, highly proliferative gliomas and conferred poor prognosis in patients with glioma (32). Activation of SMAD2 could induce migration of mouse squamous carcinoma cells and elevated levels of SMAD2 were required for induction of spindle-cell transformation and metastasis (33). Overexpression of SMAD2 was also suggested to be associated with metastasis and was correlated with poor prognosis of gastric cancers, especially diffuse-type gastric carcinoma (34). In our study SMAD2 was overexpressed and verified as a promising target gene, which was related to metastasis in gastric cancer cells. Our analysis revealed that restoring miR-155 expression attenuated the protein level of SMAD2 and therefore inhibited cell metastasis in gastric cancer.
It is known that miR-155 is processed from its precursor, BIC. Given that BIC promoter contains two predicted $\mathrm{CpG}$ islands and its methylation has been reported to be responsible for the low level or the absence of BIC expression in non-Hodgkin lymphoma cell lines (35), we demethylated two gastric cancer cell lines, and as anticipated, miR-155 was significantly upregulated. Our results demonstrate that downregulation of miR-155 in gastric cancer cells was linked to the DNA methylation. The detailed mechanisms for reduced miR-155 expression in gastric cancer cells remained to be further investigated.

In conclusion, our results identify the downregulated expression and anti-oncogenic functions of miR-155 in gastric cancer cells and shed light on its role in cell migration, invasion and adhesion. Our study might help in developing new diagnostic and therapeutic targets for the treatment of metastatic gastric cancer.

\section{Acknowledgements}

This study was supported by grants from the National Natural Science Foundation of China (no. 30872476, no. 81172324), the National High Technology Research and Development Program of China (863 Program, no. 2006AA02A301 and no. 2007AA02Z179), the Science and Technology Commission of Shanghai Municipality (no. 10jc1411100, 09DZ1950100, and 09DZ2260200), the Shanghai Key Discipline (S30204), and the Key Projects in the National Science and Technology Pillar Program of China (no. 2008BA152B03) and the Innovation Foundation for PhD Graduates (BXJ201115).

\section{References}

1. Jemal A, Bray F, Center MM, Ferlay J, Ward E and Forman D: Global cancer statistics. CA Cancer J Clin 61: 69-90, 2011.

2. Ferlay J, Shin HR, Bray F, Forman D, Mathers C and Parkin DM: GLOBOCAN $2008 \mathrm{v} 1.2$, Cancer Incidence and Mortality Worldwide: IARC CancerBase No. 10. Lyon. International Agency for Research on Cancer; Available from: http://globocan. iarc.fr. Last accessed 9/16/2011, 2010.

3. Moon YW, Jeung HC, Rha SY, et al: Changing patterns of prognosticators during 15-year follow-up of advanced gastric cancer after radical gastrectomy and adjuvant chemotherapy: a 15-year follow-up study at a single Korean institute. Ann Surg Oncol 14: 2730-2737, 2007

4. Bartel DP: microRNAs: genomics, biogenesis, mechanism, and function. Cell 116: 281-297, 2004.

5. Bartel DP: microRNAs: target recognition and regulatory functions. Cell 136: 215-233, 2009.

6. Munker R and Calin GA: microRNA profiling in cancer. Clin Sci (Lond) 121: 141-158, 2011.

7. Farazi TA, Spitzer JI, Morozov P and Tuschl T: miRNAs in human cancer. J Pathol 223: 102-115, 2011.

8. Di Leva G and Croce CM: Roles of small RNAs in tumor formation. Trends Mol Med 16: 257-267, 2010.

9. Lagos-Quintana M, Rauhut R, Yalcin A, Meyer J, Lendeckel W and Tuschl T: Identification of tissue-specific microRNAs from mouse. Curr Biol 12: 735-739, 2002.

10. Faraoni I, Antonetti FR, Cardone J and Bonmassar E: miR-155 gene: a typical multifunctional microRNA. Biochim Biophys Acta 1792: 497-505, 2009.

11. Tili E, Croce CM and Michaille JJ: miR-155: on the crosstalk between inflammation and cancer. Int Rev Immunol 28: 264-284, 2009.

12. Iorio MV, Ferracin M, Liu CG, et al: MicroRNA gene expression deregulation in human breast cancer. Cancer Res 65: 7065-7070, 2005.

13. Lee EJ, Gusev Y, Jiang J, et al: Expression profiling identifies microRNA signature in pancreatic cancer. Int J Cancer 120: 1046-1054, 2007. 
14. Bandres E, Cubedo E, Agirre X, et al: Identification by real-time PCR of 13 mature microRNAs differentially expressed in colorectal cancer and non-tumoral tissues. Mol Cancer 5: 29, 2006.

15. Nikiforova MN, Tseng GC, Steward D, Diorio D and Nikiforov YE: microRNA expression profiling of thyroid tumors: biological significance and diagnostic utility. J Clin Endocrinol Metab 93: 1600-1608, 2008.

16. Wang X, Tang S, Le SY, et al: Aberrant expression of oncogenic and tumor-suppressive microRNAs in cervical cancer is required for cancer cell growth. PLoS One 3: e2557, 2008.

17. Yanaihara N, Caplen N, Bowman E, et al: Unique microRNA molecular profiles in lung cancer diagnosis and prognosis Cancer Cell 9: 189-198, 2006.

18. Donnem T, Eklo K, Berg T, et al: Prognostic impact of MiR-155 in non-small cell lung cancer evaluated by in situ hybridization. J Transl Med 9: 6, 2011.

19. Dorsett Y, McBride KM, Jankovic M, et al: MicroRNA-155 suppresses activation-induced cytidine deaminase-mediated Myc-Igh translocation. Immunity 28: 630-638, 2008.

20. Levati L, Pagani E, Romani S, et al: microRNA-155 targets the SKI gene in human melanoma cell lines. Pigment Cell Melanoma Res 24: 538-550, 2011.

21. Levati L, Alvino E, Pagani E, et al: Altered expression of selected microRNAs in melanoma: antiproliferative and proapoptotic activity of miRNA-155. Int J Oncol 35: 393-400, 2009.

22. Chen XM, Splinter PL, O'Hara SP and LaRusso NF: A cellular micro-RNA, let-7i, regulates Toll-like receptor 4 expression and contributes to cholangiocyte immune responses against Cryptosporidium parvum infection. J Biol Chem 282 28929-28938, 2007.

23. Louafi F, Martinez-Nunez RT and Sanchez-Elsner T: MicroRNA155 targets SMAD2 and modulates the response of macrophages to transforming growth factor- $\beta$. J Biol Chem 285: 41328-41336, 2010.

24. Calin GA and Croce CM: microRNA signatures in human cancers. Nat Rev Cancer 6: 857-866, 2006.
25. Kluiver J, Haralambieva E, de Jong D, et al: Lack of BIC and microRNA miR-155 expression in primary cases of Burkitt lymphoma. Genes Chromosomes Cancer 45: 147-153, 2006.

26. Roldo C, Missiaglia E, Hagan JP, et al: microRNA expression abnormalities in pancreatic endocrine and acinar tumors are associated with distinctive pathologic features and clinical behavior. J Clin Oncol 24: 4677-4684, 2006.

27. Dahiya N, Sherman-Baust CA, Wang TL, et al: microRNA expression and identification of putative miRNA targets in ovarian cancer. PLoS One 3: e2436, 2008.

28. Wald AI, Hoskins EE, Wells SI, Ferris RL and Khan SA: Alteration of microRNA profiles in squamous cell carcinoma of the head and neck cell lines by human papillomavirus. Head Neck 33: 504-512, 2011

29. Imig J, Motsch N, Zhu JY, et al: microRNA profiling in EpsteinBarr virus-associated B-cell lymphoma. Nucleic Acids Res 39: 1880-1893, 2011

30. Miyazono K, Suzuki H and Imamura T: Regulation of TGF-beta signaling and its roles in progression of tumors. Cancer Sci 94: 230-234, 2003

31. Derynck R, Akhurst RJ and Balmain A: TGF-beta signaling in tumor suppression and cancer progression. Nat Genet 29: 117-129, 2001.

32. Bruna A, Darken RS, Rojo F, et al: High TGFbeta-Smad activity confers poor prognosis in glioma patients and promotes cell proliferation depending on the methylation of the PDGF-B gene. Cancer Cell 11: 147-160, 2007.

33. Oft M, Akhurst RJ and Balmain A: Metastasis is driven by sequential elevation of $\mathrm{H}$-ras and Smad2 levels. Nat Cell Biol 4: 487-494, 2002.

34. Shinto O, Yashiro M, Toyokawa T, et al: Phosphorylated smad2 in advanced stage gastric carcinoma. BMC Cancer 10: 652, 2010.

35. van den Berg A, Kroesen BJ, Kooistra K, et al: High expression of B-cell receptor inducible gene BIC in all subtypes of Hodgkin lymphoma. Genes Chromosomes Cancer 37: 20-28, 2003. 